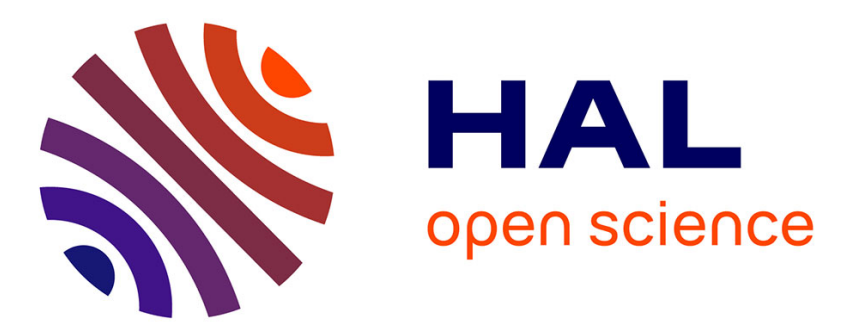

\title{
Handicaps simulés et outils numériques : un exemple d'utilisation en judo
}

Michel Calmet, Emerson Franchini, Teresa Assude

\section{To cite this version:}

Michel Calmet, Emerson Franchini, Teresa Assude. Handicaps simulés et outils numériques: un exemple d'utilisation en judo. La nouvelle revue de l'adaptation et de la scolarisation, 2014, 10.3917/nras.066.0253 . hal-01757682

\section{HAL Id: hal-01757682 \\ https://hal.science/hal-01757682}

Submitted on 5 Apr 2018

HAL is a multi-disciplinary open access archive for the deposit and dissemination of scientific research documents, whether they are published or not. The documents may come from teaching and research institutions in France or abroad, or from public or private research centers.
L'archive ouverte pluridisciplinaire HAL, est destinée au dépôt et à la diffusion de documents scientifiques de niveau recherche, publiés ou non, émanant des établissements d'enseignement et de recherche français ou étrangers, des laboratoires publics ou privés. 


\title{
Handicaps simulés et outils numériques : un exemple d'utilisation en judo
}

\author{
Calmet Michel, Université de Montpellier 1, France \\ Franchini Emerson, Université de Sao-Paulo, Brésil \\ Assude Teresa, Aix-Marseille Université, France
}

Résumé

Les outils numériques sont utilisés transversalement comme outils de recherche et outils de formation dans ce travail. L'analyse vidéo (de la prise de vue en numérique au suivi de points sur l'écran et le traitement statistique des données) a permis d'élaborer et d'évaluer un test créant un handicap simulé de "vertiges". Ce test a été réinvesti au cours d'une formation sur les Activités Physiques adaptées et Santé et 37 étudiants en $3^{\text {ème }}$ année d'études à l'Université ont pu être questionnés sur les sensations qu'ils percevaient après une technique de judo, avant et après un handicap simulé de "vertiges". Les résultats sont pluriels et suggèrent d'une part que des perceptions comme "l'amplitude", "la vue", "I'unité corporelle (corps en un ou plusieurs morceaux)" apparaissent modifiées et changent d'ordre de classement, d'autres comme "l'ouïe" et la "peur d'avoir mal" semblent moins importantes et restent en dernier rang. D'autre part, l'utilisation des outils numériques sur le terrain montre qu'ils sont à la fois outils de recherches accessibles et utilisables de façon simple (publications et mises en place/analyses de situations très proches du réel) et outils de formations (mise en place de nouveaux contenus de cours).

Mots clés : formations aux et par les outils numériques, handicaps simulés, activités physiques adaptées et santé, recherche écologique, judo.

\section{Abstract}

In this study, digital tools are transversely used as research tools and training tools. The video analysis (from recording video sequences to tracking points in the frames and the statistical treatment of data) allowed us to elaborate a test to create a simulated disability of "dizziness".

This test was reinvested in training course on Adapted Physical Activities and Health, and 37 students in their third year of studies at the University have been questioned (Condorcet's pairwise comparisons methodology) on the sensations they perceived after a judo throw, before and after a simulated disability of "dizziness".

Results are manifold and suggest firstly that perceptions as "amplitude", "view", " body unit (body in one or more pieces)" appear modified and change their rank order, and others perceptions as "hearing" and "fear of pain" seem less important and remain in same and last place. On the other hand, the use of digital tools in the field of sportive practices shown that they are both: 1) accessible tools for research and tools usable in simple ways (scientific publications and implemented or analysis very close to real situations); 2 ) training tools (introduction of new course content).

Keywords

Training with and for digital tools, simulated disabilities, adapted physical activity and health, ecological research, judo. 


\section{Introduction}

Vivre mieux et plus longtemps, recouvrer des capacités motrices et intellectuelles après un accident ou une maladie, appréhender les bouleversements qu'apportent les outils numériques figurent parmi les préoccupations d'aujourd'hui. Les recherches actuelles mettent en relation les outils numériques au service d'une meilleure vie quotidienne (domotique, diagnostics médicaux et intervention chirurgicale assistés, prothèses ou orthèses de plus en plus sophistiquées). Quelles que soient leurs études, la formation actuelle des étudiants doit prendre en compte ces phénomènes sociétaux.

Dans leurs travaux, Grayson \& Marini (1996) indiquent que des conseillers en réadaptation ont été sensibilisés à ce que pourrait être la vie quotidienne des personnes qui utilisent un fauteuil roulant. Les résultats suggèrent que les sujets ayant participé à la formation se sentent "meilleurs conseillers". A l'université de Montpellier 1, depuis 2005, les étudiants en $3^{\text {ème }}$ année de la spécialité "Activités physiques adaptées et santé" (APAS) suivent un cours de 20h intitulé "APAS et handicaps simulés, un exemple en sport de combat". Ils apprennent le judo dans le cadre de situations fondées sur le "handicap simulé" où ils sont momentanément aveugles (foulard sur les yeux), handicapés moteur (une main, une cheville, un bras attaché), sourds (boules Quiès) ou en vertige (rotations au sol) (Assude \& Calmet, 2014). Ceux qui suivent la spécialité sportive "judo" déterminent pendant des cours "informa-TIC" de 20h le système d'attaques des judokas (Calmet \& Ahmaidi, 2004; Calmet, Trezel \& Ahmaidi, 2006) ou modélisent le comportement des judokas (Calmet, Miarka \& Franchini, 2010). Par ailleurs, les récents (et de plus en plus puissants) logiciels libres et gratuits simplifient l'analyse des séquences vidéo des activités physiques (par exemple, http://www.kinovea.org/; http://www.anvil-software.org/), et les nouvelles applications sur smartphones (accéléromètres, gyroscopes) facilitent ou vont faciliter les recherches appliquées sur le terrain en judo. Ces recherches dites écologiques (proches de la réalité) viennent compléter celles effectuées dans des salles spécifiques de mesures ou dans les laboratoires.

\section{Questions de recherche et méthodologie}

Une revue de littérature montre que : i) La rééducation (avec ou sans technologies nouvelles, avec pratiques sportives adaptées) après accident ou maladie fait l'objet de nombreuses recherches ${ }^{1}$. ii) les handicaps simulés sont utilisés comme moyen de recherche (Barker \& al., 2014; Hua \& al., 2014; Pennathur, Mital, Contreras, 2001). iii) les TIC et outils numériques sont un peu utilisés en rééducation, éducation ou prévention (Arbogast \& al., 2014; Reese \& al., 2013; Hsu \& al., 2011) ou en complément des cours d'éducation physique en France (cf.: http://www.revue-eps.com/)

\footnotetext{
1 "medical rehabilitation" dans pubmed donne 67427 résultats (consulté le 17/06/2013)
} 
Constatant que peu de travaux scientifiques concernent d'une part l'utilisation des handicaps simulés comme moyen de formation dans les activités physiques et sportives (Assude \& Calmet, 2014) et d'autre part l'articulation formation-recherche par et pour les outils numériques, notre objectif est de mettre en lumière les innovations possibles concernant les formations aux APAS, des recherches écologiques, les outils numériques et les sports.

Nous prenons ici la notion de "handicap simulé" comme la simulation d'une situation de handicap et il s'agit de créer "un dispositif qui est fondé sur un certain nombre de contraintes - les handicaps simulés - qui permettent aux acteurs en formation de se rendre disponibles à une situation non usuelle et de résoudre les problèmes induits par ces contraintes." (Assude \& Calmet, 2014, p.13)

Nos questions sont alors : quels sont les effets du handicap simulé de "vertiges" sur les perceptions des étudiants à propos des vertiges pathologiques ? Comment utiliser les outils numériques pour l'analyse de ce type de situations?

Pour étudier ces questions, nous avons formulé deux hypothèses et nous avons mis en place un plan de travail. La première est que l'analyse des réponses de sujets "valides" à des questionnaires, après une situation de handicap simulé de "vertiges", peut apporter des éléments nouveaux, notamment sur les chutes, pour mieux comprendre les problèmes dus aux vertiges pathologiques. La deuxième est que les outils numériques peuvent aider les sujets à analyser les situations vécues et qu'ils sont à la fois outils de recherche et outils de formation.

Notre plan de travail s'établi donc ainsi :

1) Etablir un test sur le terrain, simulant pour quelques secondes un handicap de "vertiges".

2) Validation de ce test lors d'exercices d'entrainements de judo en étudiant des modifications de comportements après ce handicap simulé de "vertiges"

3) Mettre en place l'expérimentation : Etudes des sensations des étudiants après une projection de judo avant et après un handicap simulé de "vertiges".

4) Organiser le recueil de données en prenant en compte ce handicap de "vertiges" de quelques secondes, et travailler sur le domaine des chutes. Nous avons recueilli les sensations des judokas après une projection de judo avant et après un handicap simulé de "vertiges".

5) Comparer et interpréter les classements des réponses des étudiants.

6) Vérifier la validité de nos hypothèses dans les conclusions et dresser des perspectives. 
Les méthodes utilisées pour les points 1, 2 et 3 sont différentes. Elles sont énoncées au début de chacune des parties correspondantes.

\section{1) Etablir un test sur le terrain, créant pour quelques secondes un handicap simulé de "vertiges"}

Nous avons observé 15 étudiants en Sciences et Techniques des Activités Physiques et Sportives (STAPS) (7 filles et 8 garçons; moyenne âge 21,2 ans $\pm 0,7$; taille $175,3 \mathrm{~cm} \pm 8,3$ ) qui étaient volontaires et ont réalisé le test suivant :

1) Tâche 1 : pieds nus, station debout sur les tapis de judo, en équilibre :

a. faire trois pas en avant en démarrant pied droit, en posant le pied sur une ligne. C'est une limite entre les tapis, elle est à la fois visuelle et tactile.

b. faire demi-tour.

c. faire trois pas en avant en démarrant pied droit pour revenir au point de départ.

2) Tâche 2 : création du handicap simulé de "vertiges" :

a. réaliser le plus vite possible 5 roulades en avant, faire demi-tour et refaire à nouveau le plus vite possible 5 roulades en avant pour revenir au point de départ.

3) Tâche 3 : reprendre la station debout en équilibre après les roulades et refaire la tâche 1.

L'enregistrement est réalisé avec un appareil photo numérique (APN casio exilim EX FH20, $30 \mathrm{i} / \mathrm{s}$, VGA) posé sur un support au ras du tapis, une lampe frontale de randonnée est fixée à la cheville droite, au-dessus des malléoles des étudiants.

Avec le logiciel kinovea, les distances (projection sur l'horizontale) en pixels ont été relevées (Fig. 1):

a. pendant les trois pas : distance moyenne entre le bord de l'image et la lampe.

b. deux mesures au moment où le pied droit est en appui et le pied gauche se soulève : distance entre la lampe et le gros orteil du pied opposé.

c. deux mesures au moment où le pied droit est en appui et le pied gauche se soulève : distance entre les jonctions pouce et index des deux mains.
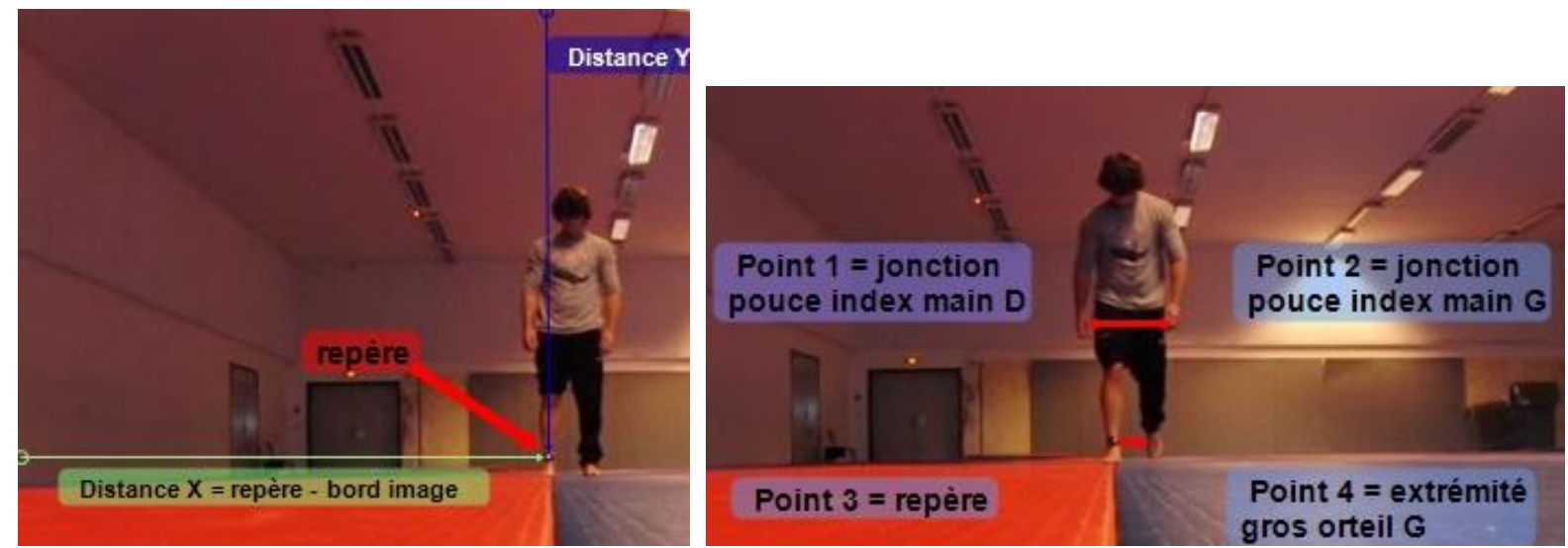
Fig. 1 : exemple de distances relevées sur les séquences vidéos

Une ANOVA (un facteur et test post-hoc de Bonferroni-Holm) a permis de vérifier si les différences entre les distances étaient significatives.

\section{Résultats}

Les étudiants sont perturbés pour marcher en équilibre tout en posant leur pied droit sur la ligne après leurs 10 roulades. L'observation visuelle est confirmée par leurs propos : "c'est pas facile"; "on dirait qu'on est saouls"; "tu m'as vu, mais j'ai pas bu".

La durée moyenne du test a été de $35,5 \mathrm{~s} \pm 3,7 \mathrm{~s}$.

\begin{tabular}{|c|c|c|c|c|c|c|c|c|}
\hline & \multicolumn{2}{|c|}{$\begin{array}{l}\text { Pose pied droit sur la } \\
\text { ligne (pixels) }\end{array}$} & \multicolumn{2}{|c|}{$\begin{array}{l}\text { Ecarts mains 1er appui } \\
\text { (pixels) }\end{array}$} & \multicolumn{2}{|c|}{$\begin{array}{l}\text { Ecarts pieds 1er appui } \\
\text { (pixels) }\end{array}$} & \multicolumn{2}{|c|}{$\begin{array}{l}\text { Différence durées } 4 \\
\text { premiers appuis (s) }\end{array}$} \\
\hline & Avant & Après & Avant & Après & Avant & Après & Avant & Après \\
\hline Moyenne & 283,8 & 282,3 & 46,1 & 76,9 & 17,3 & 20,9 & 2,6 & 2,4 \\
\hline Ecart type & 1,7 & 4,8 & 5,9 & 20,8 & 3,5 & 6,9 & 0,2 & 0,3 \\
\hline significativité & \multicolumn{2}{|c|}{ non significatif } & \multicolumn{2}{|c|}{$\begin{array}{l}F(1,28)=30,56 \\
p=6,5737 E-06\end{array}$} & \multicolumn{2}{|c|}{ non significatif } & \multicolumn{2}{|c|}{$\begin{array}{c}F(1,28)=6,5252 \\
p=0,0163608\end{array}$} \\
\hline
\end{tabular}

Tableau 1 : moyennes et écarts types des distances relevées pour les 15 sujets

Les résultats présentés dans le tableau 1 montrent que la marche :

- reste rectiligne, l'écart entre les pieds ne varie pas de façon significative.

- les bras s'écartent pour assurer l'équilibre, le temps d'exécution de la marche est réduit.

Ce handicap simulé de "vertiges" s'est caractérisé par une marche un peu précipitée avec les bras écartés. II ne dure que quelques secondes (<3s). Comme le funambule qui, en difficulté d'équilibre sur son fil, s'arrête, prend son temps pour rétablir cet équilibre nous pensions que, perturbé dans la gestion de son équilibre pour marcher, le sujet mettrait plus de temps pour exécuter la tâche $N^{\circ} 3$ que pour exécuter la tâche $N^{\circ} 1$. Nos représentations correspondaient aux travaux de Pennathur, Mital \& Contreras, (2001) où les sujets assis, en situation de handicap simulé pour manipuler finement des objets, mettaient plus de temps pour réaliser les tâches demandées. Dans nos résultats, il n'en n'est rien, les sujets ont réalisé la tâche $\mathrm{N}^{\circ} 3$ plus vite. Cependant, le test montre que l'équilibre des sujets est perturbé pendant quelques secondes et que leurs déplacements sont altérés. Ce laps de temps de perturbation est assez long pour utiliser le test lors de l'exécution de tâches courtes et de comparer l'exécution de ces tâches avant et après perturbation.

2) Validation de ce test lors d'exercices d'entrainements de judo en étudiant des modifications de comportements après ce handicap simulé de "vertiges" 
Pour automatiser l'exécution des techniques de judo, les judokas répètent tout ou partie des prises de judo (préaction, lancement, guidage; Trilles, Lacouture \& Cadière, 1990) dans des conditions variées. Un exercice appelé uchikomi consiste à n'effectuer que la première partie de la prise (préaction) et la répéter. C'est un exercice de déplacements et placement du corps avec utilisation des mains (saisies du vêtement adverse), mais cette manipulation n'est pas un geste fin "d'horloger". La vitesse, la précision et la régularité dans les déplacements, placements et saisies sont des indicateurs de réussite. Le handicap simulé de "vertiges" devrait perturber cette exécution.

Deux judokas (un haut-niveau en compétition universitaire et un débrouillé) ont participé à cette expérience et ont été filmés avec un appareil photo numérique (APN, casio exilim EX FH20, $30 \mathrm{i} / \mathrm{s}$, VGA) lors de trois tâches suivantes : exercice d'entrainement, test, exercice d'entrainement. Ils ont exécuté 10 fois la préaction de leur prise préférée, puis ils ont effectué 5 roulades pour s'éloigner de leur partenaire, ils ont fait demi-tour puis ils ont fait 5 roulades pour revenir face à leur partenaire pour refaire à nouveau 10 fois la préaction de leur prise préférée (Fig. 2).

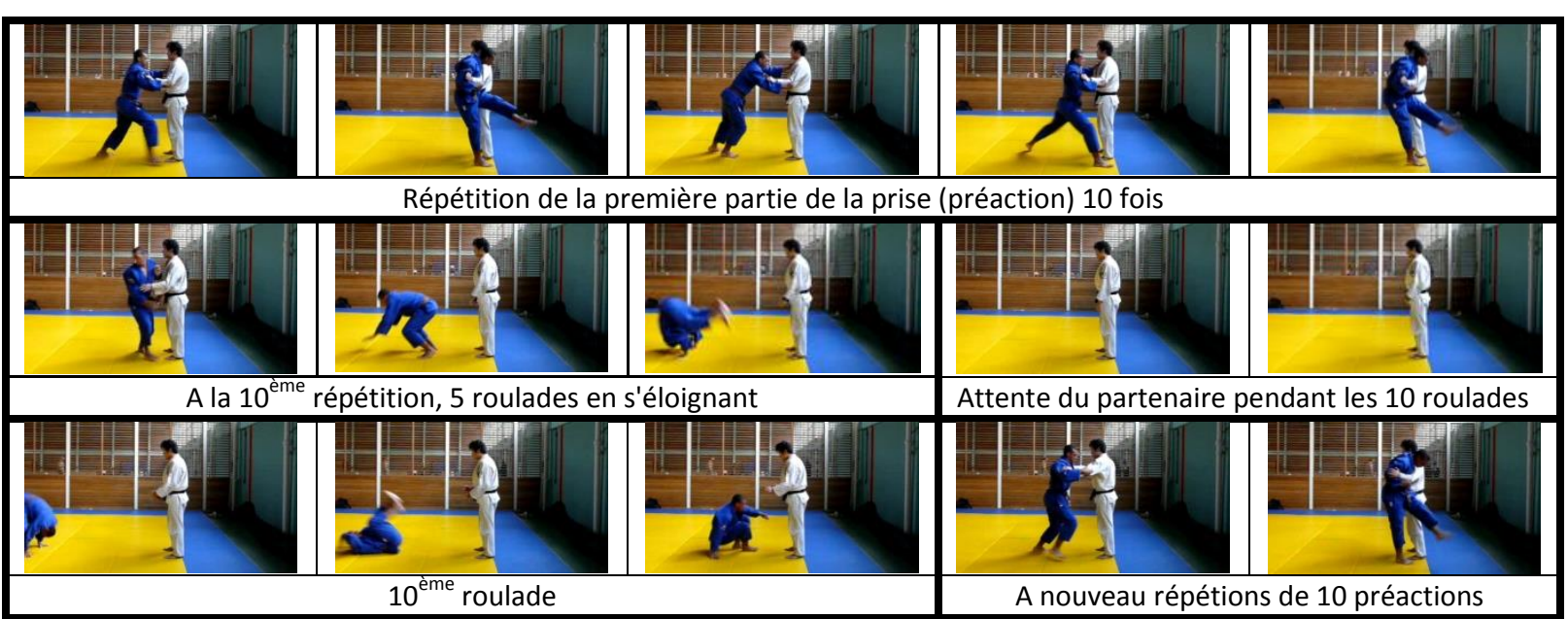

Fig. 2 : préactions de la prise préférée, roulades, reprise des préactions.

Avec le logiciel kinovea, les séquences vidéos ont permis de relever des coordonnées pour comparer les régularités dans l'exécution. Pour chacun de ces judokas, un point du corps (bassin) a été suivi (fonction tracking du logiciel). Ces coordonnées ont ensuite été exploitées dans le tableur Microsoft Excel.

Chaque vidéo est découpée en deux séquences : première et deuxième répétition. Les 2 vidéos (avant et après le handicap simulé de "vertiges") sont synchronisées sur le premier appui effectué près du partenaire lors de la répétition de la première préaction. On revient 60 images ( 2 secondes) en arrière de l'image de l'appui et on coupe ce qui précède. L'erreur relative peut être d'une image soit $0,033 \mathrm{~s}$. Nous avons utilisé le test de Student pour comparer les données de chaque athlète avant et après perturbations. Les coordonnées ont été relevées dans kinovea par la même personne, $5^{\circ}$ dan de judo, enseignant les TICE en Université depuis 2003. 
Les exercices sont spécifiques au judo et sont réalisés sur le tapis de judo, l'espace nécessaire à l'expérimentation est un couloir de $14 \mathrm{~m}^{2}(7 \mathrm{~m} \times 2 \mathrm{~m})$. L'enregistrement des séquences vidéos est fait en "situation écologique" c'est-à-dire très proches du réel, l'analyse des séquences vidéos est faite en salle informatique.

\section{Résultats}

Les deux graphiques permettent les comparaisons des répetitions pour le sujet "expert" (fig.3) et pour le sujet "débrouillé" (fig.4) avant et après le handicap simulé de "vertiges". Il s'agit pour les 2 courbes en haut de chaque graphique de la coordonnée y relevée et pour les deux courbes en bas de chaque graphique de la coordonnée $\mathrm{x}$ relevée. L'axe des abscisses est celui des images (ou du temps avec une unité de $0,033 \mathrm{~s}$ ).

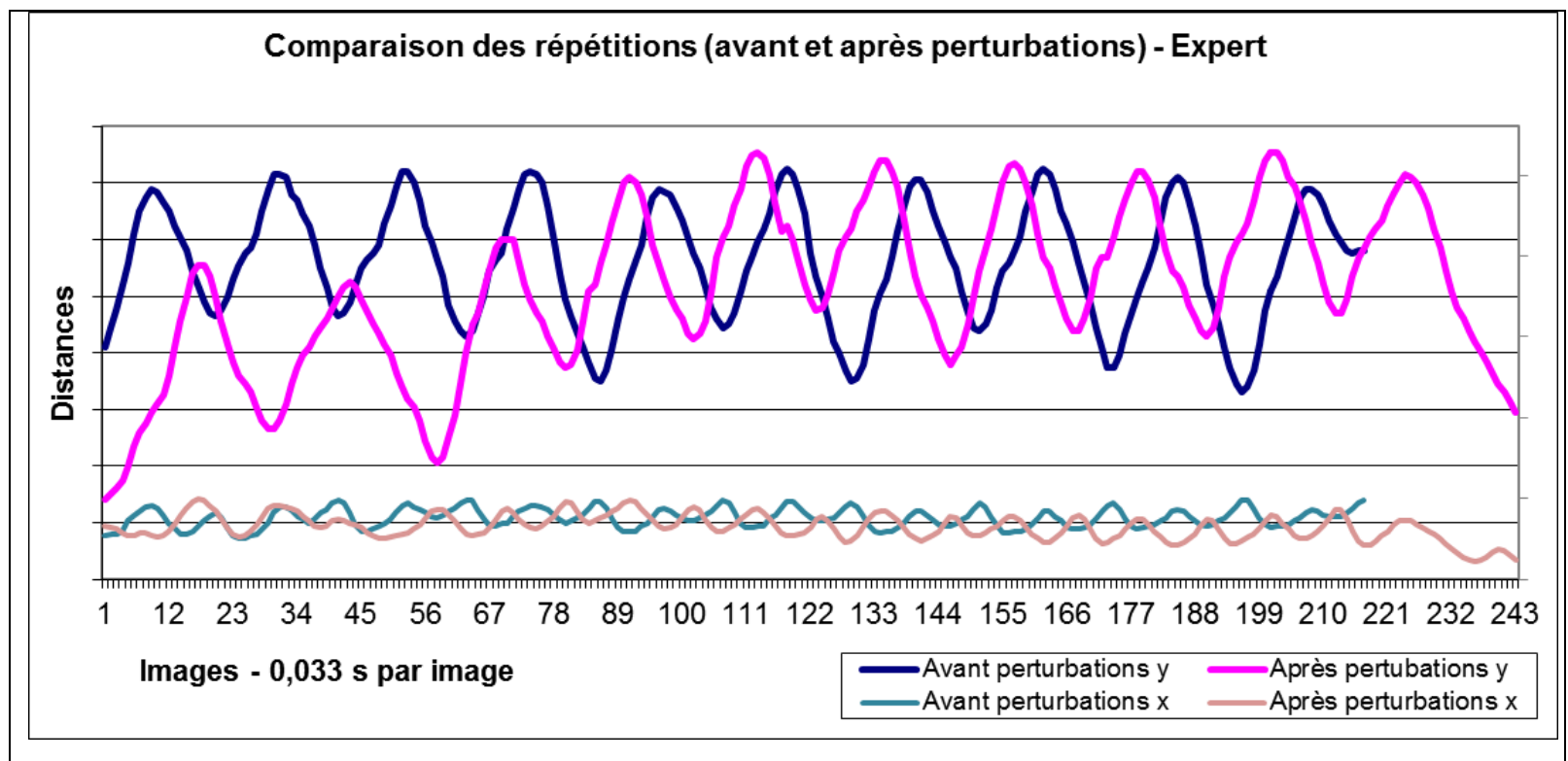

Fig 3 : comparaisons des répétitions pour le sujet expert 


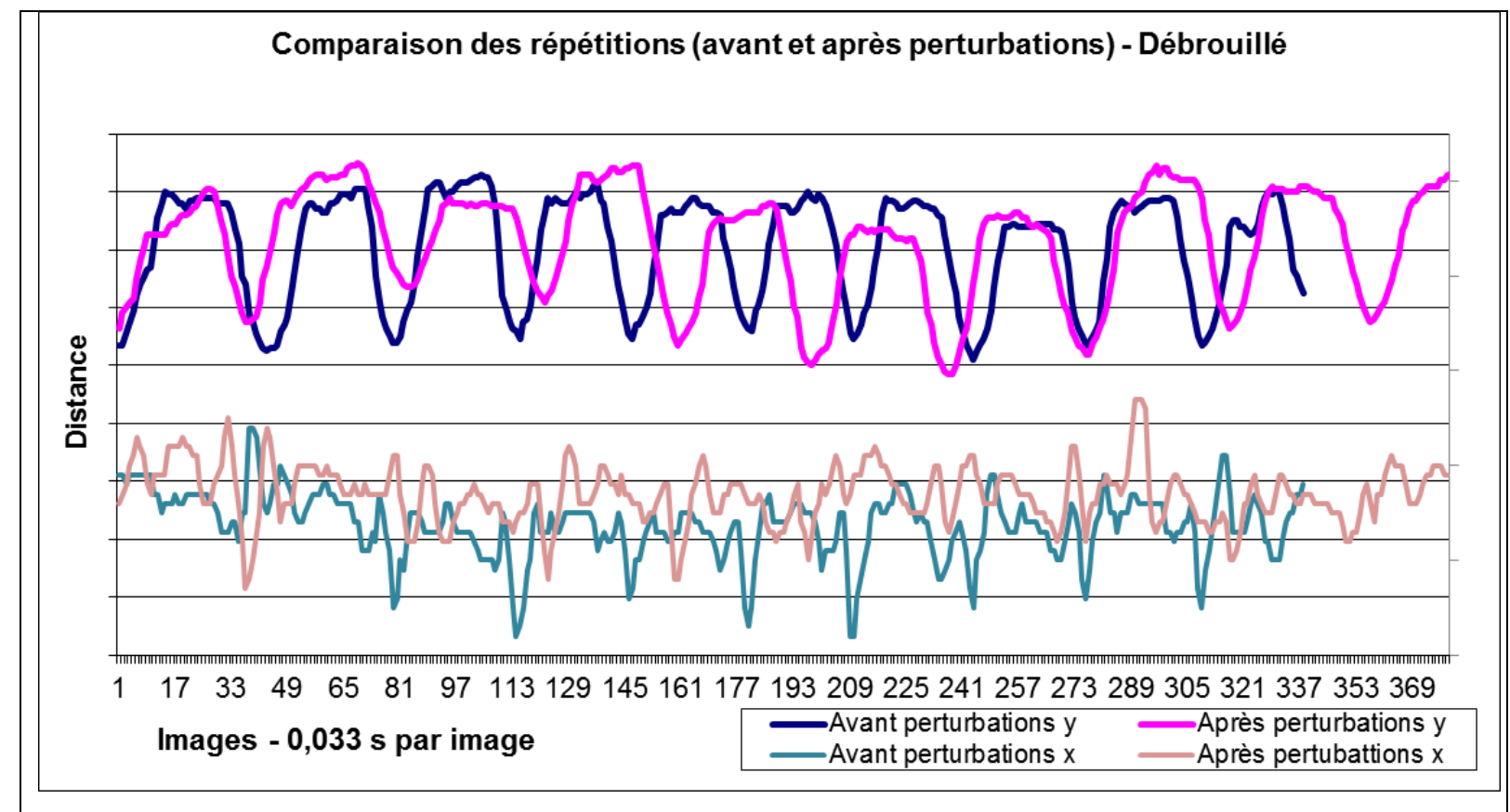

Fig 4 : comparaisons des répétitions pour le sujet débrouillé

Pour déterminer la périodicité nous avons sélectionné le point qui matérialisait une rupture de pente. Après le handicap simulé de "vertiges", le débrouillé n'a pas terminé sa dernière préaction. Nous n'avons donc retenu que les neuf premières répétitions dans notre tableau de comparaisons (Tableau 2).

\begin{tabular}{|l|c|c|c|c|c|c|c|c|}
\hline & & \multicolumn{4}{|c|}{ Points de périodicité des 9 premières répétitions } \\
\hline & $\begin{array}{c}\text { Nombre de } \\
\text { répétitions }\end{array}$ & \multicolumn{2}{|c|}{$\begin{array}{c}\text { Moyenne des } \\
\text { coordonnées y (pixel) }\end{array}$} & \multicolumn{2}{|c|}{$\begin{array}{c}\text { Moyenne des } \\
\text { coordonnées }\end{array}$ (pixel) } & \multicolumn{2}{|c|}{$\begin{array}{c}\text { Moyenne des durées de } \\
\text { périodicité (s) }\end{array}$} \\
\cline { 2 - 9 } & Avant & Après & Avant & Après & Après & Après & Avant & Après \\
\hline Expert & 10 & 10 & $322,2 \pm 2,7$ & $315,4 \pm 18,2$ & $304,1 \pm 2,4$ & $302 \pm 5$ & $0,73 \mathrm{~s} \pm 0,02$ & $0,76 \mathrm{~s} \pm 0,07$ \\
\hline Débrouillé & 10 & 9,5 & $305,4 \pm 4,2$ & $301,8 \pm 7,9$ & $179,2 \pm 1,4$ & $180,8 \pm 1,9 *$ & $1,11 \mathrm{~s} \pm 0,10$ & $1,32 \mathrm{~s} \pm 0,09 *$ \\
\hline
\end{tabular}

Tableau 2 : relevés des données lors des répétitions des préactions avant et après perturbations

\section{Discussions}

Pour des séquences courtes de ce type, le suivi et le relevé des points manuellement réalisés avec le logiciel kinovea (kinovea.org) est aisé et permet par la mise en graphique de voir la resynchronisation des répétitions. La périodicité et la synchronisation sont visibles plus aisément sur les 2 courbes en haut de chaque graphique. II y a des différences significatives $(*)$ uniquement chez le débrouillé, dans le temps de réalisation des préactions $(1,11 s \pm 0,10$ vs. $1,32 s \pm 0,09 ; p=0,004)$ et dans les coordonnées $x$ du point suivi $(p=0,04)$.

Malgré le handicap simulé de "vertiges", l'expert refait ses répétitions à l'identique (pas de différence significative) mais on peut souligner qu'à l'image 90 (soit environ $3 \mathrm{~s}$ ), après la troisième répétition, les automatismes de l'expert ont repris le dessus (Fig. 5). Si on synchronise les 2 courbes au début de la $4^{\text {ème }}$ répétition, elles sont quasiment superposées. 


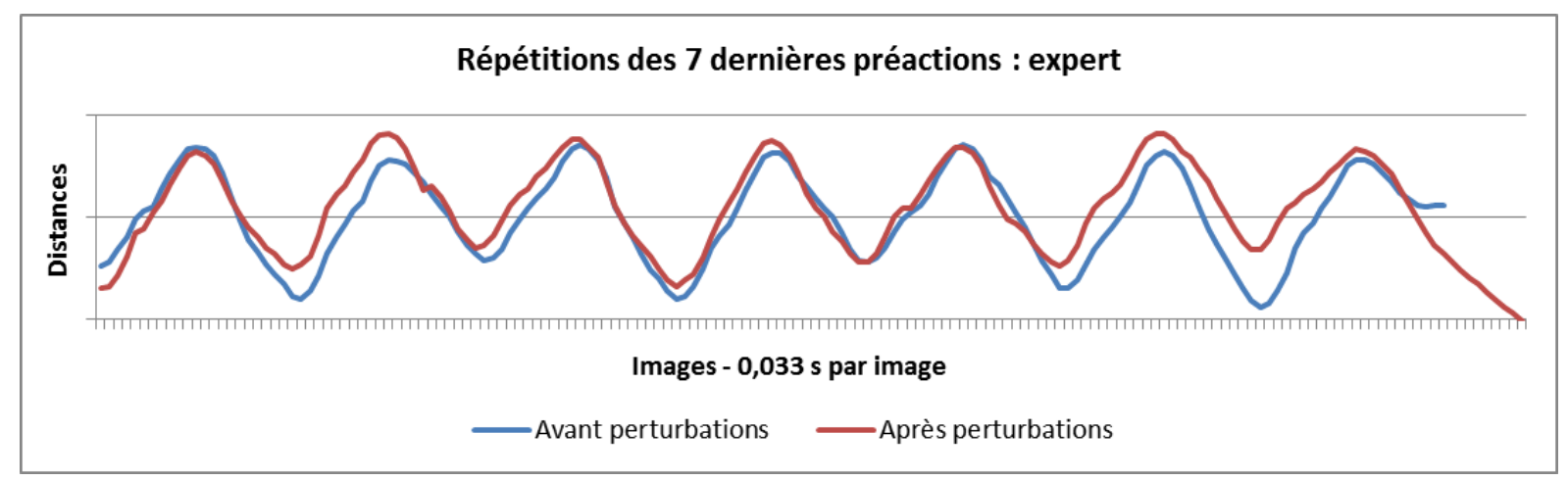

Fig. 5 : synchronisation des répétitions chez l'expert

Que ce soit avec des débutants qui marchent (lors de l'élaboration du test) ou avec des judokas (expert et débrouillé) répétant des exercices spécifiques de judo, faire ces roulades créent un handicap simulé de "vertiges". II s'estompe chez les étudiants STAPS en 3 secondes, après quelques pas ou quelques répétitions de mouvements. C'est suffisant pour expérimenter les sensations dues à une projection de judo.

\section{3) Description de l'expérimentation}

Nous allons étudier les sensations des étudiants après une projection de judo avant et après un handicap simulé de "vertiges".

a. L'étudiant "A" était projeté par un étudiant "B", puis l'étudiant "A" remplissait ses deux questionnaires 1 et 1 a, faisait 5 roulades le plus vite possible dans un sens, faisait demi-tour et revenait à son point de départ en refaisant 5 roulades le plus vite possible, il se redressait, était à nouveau projeté par l'étudiant "B", puis l'étudiant "A" remplissait ses deux questionnaires 2 et $2 \mathrm{a}$.

b. L'étudiant "B" est un judoka ceinture noire $2^{\circ}$ dan, ayant 12 ans de pratique du judo, ayant suivi les classes de spécialité judo au lycée (pôle espoir judo) et ayant choisi la spécialité judo dans ses études universitaires. La prise de judo effectuée était identique pour tous les étudiants "A", le rythme des projections ne pouvait pas le fatiguer.

c. Trois lignes d'étudiants étaient organisées, chaque ligne était gérée par 2 étudiants pour veiller à la sécurité, appeler l'étudiant "B" et veiller au remplissage des questionnaires. Toutes les 5 minutes, une nouvelle organisation de 3 lignes était mise en place. Les étudiants remplissaient l'entête de leurs 4 questionnaires avant de participer.

\section{Choix du questionnaire}

Pour recueillir les sensations des étudiants immédiatement et rapidement en environ 1'30", nous avons choisi la méthode des comparaisons par paires de Condorcet (Valade, 1995; Douglas \& Pollack, 1999). Nous avons établi 2 questionnaires à 7 items. Les étudiants ont reçu une formation à la 
méthodologie des enquêtes par comparaison par paires de 10 minutes en début de cours. Ils sont en $3^{\text {ème }}$ année d'études universitaires et la méthode des comparaisons par paire est proche de l'organisation d'un tournoi sportif dans lequel tous les participants sont opposés aux autres.

Les items ont été choisis :

a. pour le premier questionnaire en faisant référence aux sens anatomiques habituels ("toucher-contact", vue, ouie, "unité corporelle", "taille du corps") et en rajoutant des références au temps (durée) et à l'espace (amplitude).

b. le deuxième questionnaire fait référence à la difficulté de se situer dans l'espace ("Incapacité à agir", "le déséquilibre - la tête en bas", "Peur - avoir mal à l'impact au sol", "Angoisse perte des appuis au sol", "Vertige - ne plus savoir où l'on est"), "la difficulté à communiquer", "l'importance du contact".

Le nombre de 7 items permet d'avoir 21 paires ou 21 comparaisons. Cela donne de la consistance au questionnaire et permet d'avoir les réponses en 1'30" sans que la personne puisse composer un ordre de ses réponses.

37 étudiants en $3^{\text {ème }}$ année d'étude à la Faculté des Sciences du Sport, débutants en judo, ayant $17 \mathrm{~h}$ de pratique et sachant tous chuter ont accepté de suivre cette expérimentation. 


\section{4) Recueil de données}

Le recueil est organisé pour prendre en compte ce laps de temps de quelques secondes, et travailler sur le domaine des chutes.

Questionnaire 1

Qu'est-ce qui prédomine dans ce que vous avez perçu (corporellement) ?

\begin{tabular}{|l|l|l|l}
\hline NOM : & Prénom : & Année étude : & Date : \\
\hline
\end{tabular}

Pour remplir le questionnaire, il faut indiquer la préférence entre deux critères (ici : critère 3 OU critère $\mathrm{D}$ ) en entourant le choix.

\begin{tabular}{|l|l|l|}
\hline Critère 3 & Critère $\mathrm{D}$ & Dans cet exemple le critère $\mathrm{D}$ a été choisi \\
\hline
\end{tabular}

Il faut entourer votre choix pour chacune des lignes des 4 tableaux (21 choix à entourer).

\begin{tabular}{|c|c|}
\hline Durée & Amplitude \\
\hline Ouïe & Toucher, contact \\
\hline Durée & Toucher, contact \\
\hline $\begin{array}{c}\text { Toucher, contact } \\
\text { en un ou plusieurs } \\
\text { morceaux) }\end{array}$ & Amplitude \\
\hline \multicolumn{2}{|c|}{ Tableau 1} \\
\hline
\end{tabular}

\begin{tabular}{|c|c|}
\hline Ouie & Durée \\
\hline Vue & $\begin{array}{c}\text { Taille du corps, volume de la } \\
\text { tête }\end{array}$ \\
\hline $\begin{array}{c}\text { Unité corporelle (corps en } \\
\text { un ou plusieurs morceaux) }\end{array}$ & Durée \\
\hline Toucher, contact & Vue \\
\hline Vue & Ouie \\
\hline \multicolumn{2}{|c}{} \\
\hline
\end{tabular}

\begin{tabular}{|c|c|}
\hline $\begin{array}{c}\text { Unité corporelle (corps en } \\
\text { un ou plusieurs morceaux) }\end{array}$ & Ouïe \\
\hline $\begin{array}{c}\text { Taille du corps, volume de } \\
\text { la tête }\end{array}$ & Amplitude \\
\hline $\begin{array}{c}\text { Amplitude } \\
\text { la tête }\end{array}$ & $\begin{array}{c}\text { Unité corporelle (corps en } \\
\text { un ou plusieurs morceaux) }\end{array}$ \\
\hline $\begin{array}{c}\text { Taille du corps, volume de } \\
\text { Unité corporelle (corps en } \\
\text { un ou plusieurs morceaux) }\end{array}$ & $\begin{array}{c}\text { Taille du corps, volume de } \\
\text { la tête }\end{array}$ \\
\hline \multicolumn{2}{|c|}{ Tableau 3} \\
\hline
\end{tabular}

\begin{tabular}{|c|c|}
\hline Toucher, contact & $\begin{array}{c}\text { Unité corporelle (corps en } \\
\text { un ou plusieurs morceaux) }\end{array}$ \\
\hline Amplitude & Ouïe \\
\hline Toucher, contact & $\begin{array}{c}\text { Taille du corps, volume de la } \\
\text { tête }\end{array}$ \\
\hline Durée & $\begin{array}{c}\text { Taille du corps, volume de la } \\
\text { tête }\end{array}$ \\
\hline Vue & Amplitude \\
\hline Vue & Durée \\
\hline \multicolumn{2}{|c|}{ Tableau 4} \\
\hline
\end{tabular}




\section{Questionnaire 2}

Qu'avez-vous le plus ressenti (psychologiquement) quand vous avez été projeté ?

\begin{tabular}{|l|l|l|l|}
\hline NOM : & Prénom : & Année étude : & Date : \\
\hline
\end{tabular}

Pour remplir le questionnaire, il faut indiquer la préférence entre deux critères (ici : critère 3 OU critère $\mathrm{D}$ ) en entourant le choix.

\begin{tabular}{|l|l|l|}
\hline Critère 3 & Critère $D$ & Dans cet exemple le critère $\mathrm{D}$ a été choisi \\
\hline
\end{tabular}

Il faut entourer votre choix pour chacune des lignes des 4 tableaux (21 choix à entourer).

\begin{tabular}{|c|c|}
\hline $\begin{array}{c}\text { Peur (avoir mal à l'impact } \\
\text { au sol) }\end{array}$ & $\begin{array}{c}\text { Le déséquilibre (passage la } \\
\text { tête en bas) }\end{array}$ \\
\hline $\begin{array}{c}\text { Angoisse (en l'air, à la } \\
\text { perte des appuis au sol) }\end{array}$ & L'importance du contact \\
\hline $\begin{array}{c}\text { Peur (avoir mal à l'impact } \\
\text { au sol) }\end{array}$ & L'importance du contact \\
\hline $\begin{array}{c}\text { L'importance du contact } \\
\text { Incapacité à agir }\end{array}$ & $\begin{array}{c}\text { Le déséquilibre (passage la } \\
\text { tête en bas) }\end{array}$ \\
\hline Difficultés à communiquer \\
\hline Tableau 1
\end{tabular}

\begin{tabular}{|c|c|}
\hline $\begin{array}{c}\text { Angoisse (en l'air, à la perte } \\
\text { des appuis au sol) }\end{array}$ & $\begin{array}{c}\text { Peur (avoir mal à l'impact au } \\
\text { sol) }\end{array}$ \\
\hline Difficultés à communiquer & $\begin{array}{c}\text { Vertige (ne plus savoir où } \\
\text { l'on est) }\end{array}$ \\
\hline Incapacité à agir & $\begin{array}{c}\text { Peur (avoir mal à l'impact au } \\
\text { sol) }\end{array}$ \\
\hline L'importance du contact & Difficultés à communiquer \\
\hline Difficultés à communiquer & $\begin{array}{c}\text { Angoisse (en l'air, à la perte } \\
\text { des appuis au sol) }\end{array}$ \\
\hline \multicolumn{2}{|c|}{ Tableau 2} \\
\hline
\end{tabular}

\begin{tabular}{|c|c|}
\hline Incapacité à agir & $\begin{array}{c}\text { Angoisse (en l'air, à la } \\
\text { perte des appuis au sol) }\end{array}$ \\
\hline $\begin{array}{c}\text { Vertige (ne plus savoir où } \\
\text { l'on est) }\end{array}$ & $\begin{array}{c}\text { Le déséquilibre (passage la } \\
\text { tête en bas) }\end{array}$ \\
\hline $\begin{array}{c}\text { Le déséquilibre (passage la } \\
\text { tête en bas) }\end{array}$ & Incapacité à agir \\
\hline $\begin{array}{c}\text { Vertige (ne plus savoir où } \\
\text { l'on est) }\end{array}$ & $\begin{array}{c}\text { Angoisse (en l'air, à la } \\
\text { perte des appuis au sol) }\end{array}$ \\
\hline Incapacité à agir & $\begin{array}{c}\text { Vertige (ne plus savoir où } \\
\text { l'on est) }\end{array}$ \\
\hline \multicolumn{2}{|c|}{ Tableau 3} \\
\hline
\end{tabular}

\begin{tabular}{|c|c|}
\hline L'importance du contact & Incapacité à agir \\
\hline $\begin{array}{c}\text { Le déséquilibre (passage la } \\
\text { tête en bas) }\end{array}$ & $\begin{array}{c}\text { Angoisse (en l'air, à la perte } \\
\text { des appuis au sol) }\end{array}$ \\
\hline L'importance du contact & $\begin{array}{c}\text { Vertige (ne plus savoir où } \\
\text { l'on est) }\end{array}$ \\
\hline $\begin{array}{c}\text { Peur (avoir mal à l'impact au } \\
\text { sol) }\end{array}$ & $\begin{array}{c}\text { Vertige (ne plus savoir où } \\
\text { l'on est) }\end{array}$ \\
\hline Difficultés à communiquer & $\begin{array}{c}\text { Le déséquilibre (passage la } \\
\text { tête en bas) }\end{array}$ \\
\hline Difficultés à communiquer & $\begin{array}{c}\text { Peur (avoir mal à l'impact au } \\
\text { sol) }\end{array}$ \\
\hline \multicolumn{2}{|c|}{ Tableau 4} \\
\hline
\end{tabular}




\section{5) Classements des réponses des étudiants, résultats}

Nous avons recueilli 37 réponses pour chacun des deux questionnaires. Les deux tableaux suivants montrent les rangs des réponses pour chaque item avant et après le handicap simulé de "vertiges".

\begin{tabular}{|l|c|c|}
\hline \multicolumn{3}{|c|}{ Questionnaire sur les sens } \\
\hline \multicolumn{1}{|c|}{ Items } & $\begin{array}{c}\text { Classement } \\
\text { avant }\end{array}$ & $\begin{array}{c}\text { Classement } \\
\text { après }\end{array}$ \\
\hline Amplitude & 1 & 3 \\
\hline Toucher, contact & 2 & 3 \\
\hline $\begin{array}{l}\text { Taille du corps, } \\
\text { volume de la tête }\end{array}$ & 3 & 3 \\
\hline Vue & 4 & 1 \\
\hline $\begin{array}{l}\text { Unité corporelle } \\
\text { (corps en un ou } \\
\text { plusieurs morceaux) }\end{array}$ & 5 & 3 \\
\hline Durée & 6 & 7 \\
\hline Ouïe & 7 & 3 \\
\hline
\end{tabular}

\begin{tabular}{|l|c|c|}
\hline \multicolumn{2}{|c|}{ Questionnaire sur les ressentis psychologiques } \\
\hline \multicolumn{1}{|c|}{ Items } & $\begin{array}{c}\text { Classement } \\
\text { avant }\end{array}$ & $\begin{array}{c}\text { Classement } \\
\text { après }\end{array}$ \\
\hline $\begin{array}{l}\text { Le déséquilibre } \\
\text { (passage la tête en bas) }\end{array}$ & 1 & 2 \\
\hline $\begin{array}{l}\text { L'importance du } \\
\text { contact }\end{array}$ & 2 & 5 \\
\hline Incapacité à agir & 3 & 3 \\
\hline $\begin{array}{l}\text { Angoisse (en l'air, à la } \\
\text { perte des appuis au sol) }\end{array}$ & 4 & 5 \\
\hline $\begin{array}{l}\text { Vertige (ne plus savoir } \\
\text { où l'on est) }\end{array}$ & 5 & 1 \\
\hline $\begin{array}{l}\text { Difficultés à } \\
\text { communiquer }\end{array}$ & 6 & 4 \\
\hline $\begin{array}{l}\text { Peur (avoir mal à } \\
\text { l'impact au sol) }\end{array}$ & 7 & 7 \\
\hline
\end{tabular}

Nous comparons ces données sous la forme d'un tableau :

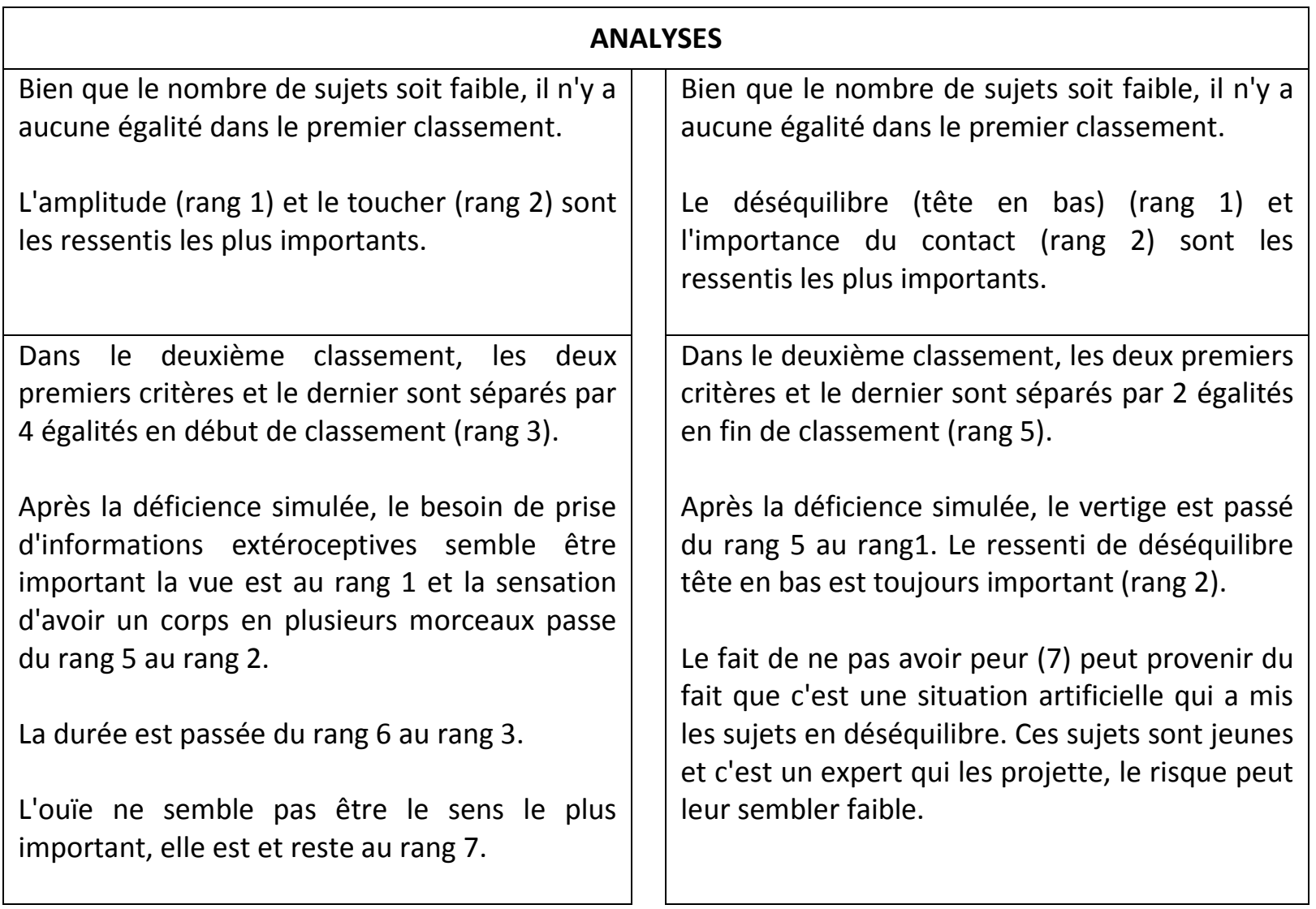




\section{6) Conclusions}

L'étude du deuxième questionnaire apporte des informations intégrables dans la formation des étudiants notamment pour mieux comprendre les problèmes dus aux vertiges pathologiques :

- L'item "Importance du contact" est passé du rang 2 au rang 5. Pourtant, de nombreux sujets (judoka "A") racontent que dès que le judoka "B" les saisit pour les projeter, ils se sentent en sécurité. Ceci rappelle les propos des sujets âgés traumatisés après une chute, qui demandent à être tenus fortement quand ils se déplacent.

- L'item "Peur (avoir mal à l'impact au sol)" est et est resté en $7^{\text {ème }}$ position. Tous les étudiants ont appris à chuter et sont projetés par un expert. Savoir chuter et par extension savoir aller au sol sans se faire mal est une compétence accessible à tous les âges. Des gestes simples doivent être appris à tous les âges : ne pas lutter et tenter de conserver une station debout jusqu'au bout quand on ressent des vertiges, il vaut mieux abaisser le centre de gravité (s'accroupir, s'asseoir).

- Les classements des items "Le déséquilibre (passage la tête en bas)" ( $1^{\text {ère }}$ position puis $2^{\text {ème }}$ ) et "Vertige (ne plus savoir où l'on est)" ( $5^{\text {ème }}$ position puis $1^{\text {ère }}$ ) montrent leurs importances. Ils serviront de base à la construction de nouvelles situations pédagogiques pour apprendre ou continuer à se repérer après des passages "la tête en bas" ou après des situations de vertiges.

- Le discours des étudiants vient compléter les questionnaires. Grâce à ce handicap simulé de "vertiges", les étudiants découvrent et comprennent les difficultés qu'ont les patients atteints de vertiges pathologiques, ils internalisent et précisent qu'ils intègreront leurs sensations dans leurs gestes et attitudes professionnelles avec ces patients.

Comme nous l'avons vu, le test repose sur le principe de créer une perturbation dans l'exécution de tâches. Par ailleurs, nous avons commencé des études avec d'autres situations d'entrainements en judo et des perturbations à partir d'actions de défenses du partenaire. Les résultats montrent que les experts retrouvent leur régularité plus vite que les débrouillés. Ces études vont dans le même sens que ceux présentés ici.

En ce qui concerne l'apport des outils numériques pour la recherche, il faut souligner d'abord la puissance (toujours plus importante), la variété (toujours plus grande) et le coût (toujours plus bas) des outils numériques permettant des relevés de données en "situation écologique", c'est-à-dire très proches du réel. L'analyse vidéo sur le terrain à des fins d'expertise des "intelligences spatiale et kinesthésique" (Gardner, 1998) devrait être facilitée avec l'arrivée des APN haute définition (1920 x 1080) et des caméras embarquées. Les tableurs et outils statistiques prennent ensuite le relais pour détailler les résultats. Dans notre étude, les données relevées montrent des régularités fines, pour 10 
répétitions l'expert exécute ses répétitions avec une moyenne de 0,73 s et un écart type de 0,02 s. Avec ces outils numériques, il devient aisé de faire des mesures précises au cours d'entrainements sportifs et d'avoir un nombre important de sujets et des groupes de sujets variés. De plus, pour traiter un nombre de sujets plus important en "situation écologique" ou relever plusieurs points spécifiques, nous plaçons des marqueurs (lampe de randonnées, bandeaux luminescents, bandes adhésives fluo) et nous travaillons en faible luminosité ce qui n'est pas sans importance dans le recueil des données.

En ce qui concerne l'apport des outils numériques pour la formation, deux exemples peuvent être signalés :

- Les possibilités de suivi de points dans l'image (tracking) ont permis la création d'un module "couplage vidéo-graphique" dans le cours "Analyse de séquences vidéos - Systèmes d'aides multimédias interactifs" pour les étudiants de Master 1. Ce couplage est réalisé en 2D avec des données issues d'une seule caméra.

- Les questionnaires utilisant la méthode des comparaisons par paires ont fait l'objet d'un TD "Traitement d'enquêtes - tableurs niveau avancé" pour les étudiants de Master 1.

\section{7) Perspectives}

Nous avons plusieurs pistes possibles à explorer prochainement. Nous en indiquons ici quelquesunes. La première consiste à changer quelques éléments de notre dispositif et voir les effets de ces changements sur les perceptions des étudiants. Par exemple, pour la création du handicap simulé de "vertiges", une autre possibilité était de faire tourner le judoka "A" sur place dans l'axe vertical. Cela aurait permis de faire l'expérimentation en salle de mesures, mais à l'essai avec les étudiants sur le tapis de judo, les roulades pourraient peut-être créer une sensation de vertige beaucoup plus forte. Une deuxième piste, dans une perspective psychomotrice, serait d'analyser pourquoi, lors de l'élaboration du test, les sujets ont effectué la deuxième tâche (marche normale) plus rapidement alors que les judokas débrouillé et expert lors des répétitions des préactions ont mis respectivement plus de temps ou le même temps pour réaliser leur deuxième tâche.

Les prochains recueils de données permettront, avec l'appui de psychologues, de :

- Affiner la sensation de corps éclaté en plusieurs morceaux.

- Mieux cibler les critères et expliciter les résultats, distribuer un seul questionnaire avec les 7 critères suivants : Durée / Amplitude; Toucher, contact; Vue; Passage tête en bas; Peur d'avoir mal à l'impact; Diminution des possibilités d'action; Difficulté à communiquer.

- Affiner les raisons du changement sur la notion de durée (la projection semble-t-elle plus longue ou plus courte?) 
D'un point de vue de l'usage des outils numériques, nous envisageons de :

- Relever des données avec 2 caméras pour obtenir des trajectoires en 3D.

- Développer une application "Questionnaires comparaisons par paires" à remplir en ligne sur tablettes ou sur smartphone.

\section{Références}

Arbogast H, Burke RV, Muller V, Ruiz P, Knudson MM, Upperman JS., 2014, Randomized controlled trial to evaluate the effectiveness of a video game as a child pedestrian educational tool; J Trauma Acute Care Surg, 76(5):1317-21

Assude, T., Calmet, M., 2014, Handicaps simulés : un outil de formation, Les Sciences de l'Education Pour l'Ere Nouvelle, vol. 47, $\mathrm{n}^{\circ} 1,11-36$

Barker A., Musso MW., Jones GN., Roid G., Gouvier D., 2014, Unreliable block span reveals simulated intellectual disability on the stanford-binet intelligence scales-fifth edition; Appl Neuropsychol Adult, 21(1):51-9

Calmet, M., 2007, Developing ecological research in judo, Perceptual and motor skills, 105, 646-648

Calmet, M., Ahmaidi, S., 2004, Survey of the advantages obtained by judoka in competition according to their level of practice, Perceptual and Motor Skills. 99: 284-290

Calmet, M., Miarka, B., Franchini, E., 2010, Modeling of grasps in judo competition contests, International Journal of Performance Analysis in Sports, 10, 229-240.

Calmet, M., Trezel, N., Ahmaidi, S., 2006, Survey of the system of attacks of regional - interregional level's judoka, Perceptual and motor skills, 103, 835-840

Douglas, B., Pollack, R., 1999, La logique du choix collectif in Les Mathématiques sociales, Dossier HS Pour la Science, juillet 1999, 82-89

Gardner, H,. 1998, Les intelligences multiples, Sciences humaines 86 bis, 6-7

Grayson E, Marini I., 1996, Simulated disability exercises and their impact on attitudes toward persons with disabilities; Int J Rehabil Res, 19(2):123-31.

Hsu JK, Thibodeau R, Wong SJ, Zukiwsky D, Cecile S, Walton DM., 2011, A "Wii" bit of fun: the effects of adding Nintendo Wii $\left({ }^{\oplus}\right)$ Bowling to a standard exercise regimen for residents of long-term care with upper extremity dysfunction; Physiother Theory Pract, 27(3):185-93.

Hua H., Emilsson M., Ellis R., Widén S., Möller C., Lyxell B., 2014, Cognitive skills and the effect of noise on perceived effort in employees with aided hearing impairment and normal hearing; Noise Health, 16(69):79-88. 
Pennathur A1, Mital A, Contreras LR., 2001, Performance reduction in finger amputees when reaching and operating common control devices: a pilot experimental investigation using a simulated finger disability; J Occup Rehabil, 11(4):281-90.

Reese RM., Jamison R., Wendland M., Fleming K., Braun MJ., Schuttler JO., Turek J., 2013, Evaluating interactive videoconferencing for assessing symptoms of autism; Telemed J E Health, 19(9):671-7

Trilles, F., Lacouture, P., Cadière, R. (1990). Analyse de différents styles d'une projection de Judo (uchi mata). Perspectives didactiques à partir d'une approche mécanique. Revue Science et Motricité, n010, pp.33-43

Valade B, 1995, Marquis de Condorcet, CD ROM Encyclopédia Universalis, Req. : Condorcet. 